\title{
Gut permeability, inflammation, and bone density across the menopause transition
}

\author{
Albert Shieh, ${ }^{1}$ Marta Epeldegui, ${ }^{2}$ Arun S. Karlamangla, ${ }^{1}$ and Gail A. Greendale ${ }^{1}$ \\ 'Division of Geriatrics, Department of Medicine, and 'Department of Obstetrics and Gynecology, David Geffen School of \\ Medicine, UCLA, Los Angeles, California, USA.
}

BACKGROUND. Inflammation is implicated in many aging-related disorders. In animal models, menopause leads to increased gut permeability and inflammation. Our primary objective was to determine if gut permeability increases during the menopause transition (MT) in women. Our exploratory objectives were to examine whether greater gut permeability is associated with more inflammation and lower bone mineral density (BMD).

METHODS. We included 65 women from the Study of Women's Health Across the Nation (SWAN). Key measures were markers of gut permeability (gut barrier dysfunction, fatty acid binding protein 2 [FABP2]) and immune activation secondary to gut microbial translocation (LPS binding protein [LBP], soluble CD14 [sCD14]), inflammation (high-sensitivity CRP), and lumbar spine (LS) or total hip (TH) BMD.

RESULTS. In our primary analysis, FABP2, LBP, and sCD14 increased by $22.8 \%(P=0.001), 3.7 \%$ ( $P$ $=0.05)$, and $8.9 \%(P=0.0002)$, respectively, from pre- to postmenopause. In exploratory, repeated measures, mixed-effects linear regression (adjusted for BMI, age at the premenopausal visit, race/ ethnicity, and study site), greater gut permeability was associated with greater inflammation, along with lower LS and TH BMD.

CONCLUSION. Gut permeability increases during the MT. Greater gut permeability is associated with more inflammation and lower BMD. Future studies should examine the longitudinal associations of gut permeability, inflammation, and BMD.

FUNDING. Funding for this research was provided by NIH, Department of Health and Human Services, through the National Institute on Aging, National Institute of Nursing Research, and NIH Office of Research on Women's Health (U01NR004061, U01AG012505, U01AG012535, U01AG012531, U01AG012539, U01AG012546, U01AG012553, U01AG012554, and U01AG012495).

Conflict of interest: The authors have declared that no conflict of interest exists.

Copyright: () 2020, American Society for Clinical Investigation.

Submitted: October 7, 2019

Accepted: December 4, 2019

Published: December 12, 2019

Reference information: /CI Insight. 2020;5(1):e134092.

https://doi.org/10.1172/jici.

insight.134092.

\section{Introduction}

Inflammation contributes to the pathogenesis of numerous medical disorders that commonly affect older adults, including osteoporosis, diabetes mellitus, cardiovascular disease, and dementia (1-10); this is often referred to as inflammaging $(3,11)$. Beyond chronological aging, the postmenopausal state may independently contribute to greater inflammation in older women. In animal models, chemical or surgical menopause leads to downregulation of epithelial junction proteins, gut barrier dysfunction, and increased gut permeability (12). This, in turn, permits the translocation of microbes from the intestinal lumen into the subepithelial space, triggering immune cells to produce proinflammatory cytokines (12). Whether an increase in gut permeability accompanies the menopause transition (MT) in humans is uncertain. If gut permeability does increase, whether it is associated with inflammation and end-organ manifestations is also unknown.

The overarching goals of this pilot study were to determine if gut permeability increases during the MT and, if such an increase were confirmed, to explore whether gut permeability is associated with inflammation and bone mineral density (BMD). In this report, subsequent use of the term "gut permeability" refers to its indirect assessment using these blood markers. We used bone as a model end-organ system because increased gut permeability mediates inflammation and hypogonadal BMD loss in rodents, and probiotics that reduce gut permeability (12) or inflammation (13-15) can decrease bone resorption (12-15) and prevent bone loss $(12,15)$. Our primary hypothesis was that gut permeability increases from pre- to 
Table 1. Descriptive statistics in pre- versus postmenopause for analytic sample $(n=65)$ : SWAN

\begin{tabular}{|c|c|c|c|}
\hline & Premenopause $^{A}$ & Postmenopause $^{\mathrm{B}}$ & $P$ value \\
\hline Age (years) ${ }^{c}$ & 49.9 (1.9) & $57.5(1.8)$ & $<0.0001$ \\
\hline $\mathrm{BMI}\left(\mathrm{kg} / \mathrm{m}^{2}\right)^{\mathrm{C}}$ & $27.6(5.6)$ & $28.9(6.1)$ & $<0.001$ \\
\hline \multicolumn{4}{|l|}{ Ovarian function markers } \\
\hline $\mathrm{E} 2(\mathrm{pg} / \mathrm{mL})^{\mathrm{D}}$ & $51.7(32.3,86.6)$ & $15.5(10.7,20.1)$ & $<0.0001$ \\
\hline $\mathrm{FSH}(\mathrm{mlU} / \mathrm{mL})^{\mathrm{D}}$ & $14.8(10.0,21.8)$ & $84.3(64.1,108.2)$ & $<0.0001$ \\
\hline $\operatorname{LBP}(n g / m L)^{c}$ & $5892(1,139)$ & $6112(1,037)$ & 0.05 \\
\hline $\mathrm{sCD14}(\mathrm{ng} / \mathrm{mL})^{\mathrm{c}}$ & $948(176.2)$ & $1032(212)$ & 0.0002 \\
\hline \multicolumn{4}{|l|}{ Inflammation } \\
\hline hs-CRP (mg/L) ${ }^{D}$ & $1.4(0.5,3.3)$ & $1.6(0.8,3.3)$ & 0.06 \\
\hline \multicolumn{4}{|l|}{ Bone mineral density } \\
\hline
\end{tabular}

postmenopause. Our exploratory hypothesis was that greater gut permeability is associated with greater inflammation (assessed by high-sensitivity CRP [hs-CRP]) and lower BMD (assessed by dual-energy X-ray absorptiometry [DXA]).

The parent study for this pilot was the Study of Women's Health Across the Nation (SWAN), a multiracial/ethnic, longitudinal cohort study of the MT. We obtained 2 stored plasma samples for each study participant from the SWAN Repository: 1 from premenopause (operationalized as 3-5 years before the final menstrual period [FMP]), and 1 from postmenopause (operationalized as $3-5$ years after the FMP). To assess gut permeability, we assayed indirect markers of gut barrier dysfunction (fatty acid binding protein 2 [FABP2]) and immune activation secondary to gut microbial translocation (LPS binding protein [LBP], soluble CD14 [sCD14]). FABP2 is considered a marker of gut epithelial cellular dysfunction because it is expressed by enterocytes and released into the circulation when gut epithelial cells are damaged (16, 17). LBP and SCD14 are deemed markers of immune activation secondary to gut microbial translocation because they are produced by hepatocytes and monocytes/macrophages, respectively, in response to LPS (a product of gram-negative bacterial, of which the gut is the predominant source) $(18,19)$.

\section{Results}

Participant characteristics in pre- to postmenopause. We included a total of 65 women. At the premenopausal visit, subjects were, on average, 49.9 years of age and 3.8 years before their FMP. The racial/ethnic composition of the study sample was as follows: 35 (57.4\%) white, $10(16.4 \%)$ Chinese, 8 (13.1\%) black, and 8 (13.1\%) Japanese. At the postmenopausal visit, mean age was 57.7 years, and mean time after the FMP was 4.0 years (Table 1$)$.

Change in gut permeability during the MT. From pre- to postmenopause, median estradiol (E2) decreased from 51.7 to $15.5 \mathrm{pg} / \mathrm{mL}(P<0.0001$, Wilcoxon signed-rank test), and follicle stimulating hormone (FSH) increased from 14.8 to $84.3 \mathrm{mIU} / \mathrm{mL}(P<0.0001$, Wilcoxon signed-rank test) (Table 1). E2 and FSH had skewed distributions and, therefore, were log-transformed for analyses. During the same period, FABP2, LBP, and sCD14 increased from 1298 to $1595 \mathrm{pg} / \mathrm{mL}, 5892$ to $6112 \mathrm{ng} / \mathrm{mL}$, and 948 to $1032 \mathrm{ng} / \mathrm{mL}$, respectively.

In our primary analysis, we used the paired $t$ test at a 2 -sided $\alpha$ of 0.05 to determine if the rise in each gut permeability marker was statistically significant. Using this approach, the increases in FABP2 $(P=0.001), \operatorname{LBP}(P=0.05)$, and $\operatorname{sCD} 14(P=0.0002)$ were considered statistically significant. We further 
Table 2. Associations of E2 or FSH with gut permeability ${ }^{A}$

\begin{tabular}{|c|c|c|}
\hline & $\begin{array}{l}\text { Increment in FABP2 }(\mathrm{pg} / \mathrm{mL}) \text {, LBP }(\mathrm{ng} / \mathrm{mL}) \text {, or sCD14 }(\mathrm{ng} / \mathrm{mL}) \text { per 2-fold } \\
\text { decrement in E2 or 2-fold increment in FSH }(95 \% \mathrm{Cl})^{\mathrm{B}}\end{array}$ & $P$ value \\
\hline \multicolumn{3}{|l|}{ FABP2 } \\
\hline E2 & $77(10,143)$ & 0.02 \\
\hline FSH & $113(47,179)$ & 0.001 \\
\hline \multicolumn{3}{|l|}{ LBP } \\
\hline E2 & $33(-41,110)$ & 0.3 \\
\hline FSH & $21(-55,98)$ & 0.5 \\
\hline \multicolumn{3}{|l|}{ sCD14 } \\
\hline E2 & $28(12,44)$ & 0.001 \\
\hline FSH & $34(19,50)$ & $<0.0001$ \\
\hline \multicolumn{3}{|c|}{$\begin{array}{l}\text { ACut permeability assessed by FABP2 (barrier dysfucntion), LBP, and sCD14 (immune activation secondary to gut } \\
\text { microbial translocation). }{ }^{B} \text { Associations assessed by repeated measured, mixed-effect linear regression with FABP, } \\
\text { LBP, or sCD14 as continuous outcome variable and E2 or FSH (log transformed) as continuous primary predictors } \\
\text { (each outcome/primary predictor pair modeled separately). Analyses were adjusted for age at premenopausal time } \\
\text { point, BMI, race/ethnicity, and Study of Women's Health Across the Nation (SWAN) study site. E2, estradiol; FSH, } \\
\text { follicle stimulating hormone. }\end{array}$} \\
\hline
\end{tabular}

examined the associations of these markers with E2 and FSH (log transformed), using repeated measures, mixed-effects linear regression (Table 2). Adjusted for chronological age at the premenopausal visit, race/ ethnicity, BMI, and study site, lower E2 and greater FSH (tested separately) were associated with greater FABP2 and sCD14. Each 50\% decrement in E2 was associated with $77 \mathrm{pg} / \mathrm{mL}$ greater FABP2 $(P=0.02)$ and $28 \mathrm{ng} / \mathrm{mL}$ greater sCD14 $(P=0.001)$. Analogously, each 2-fold increment in FSH was associated with $113 \mathrm{pg} / \mathrm{mL}$ greater FABP2 $(P=0.001)$ and $34 \mathrm{ng} / \mathrm{mL}$ greater $\mathrm{sCD} 14(P<0.0001)$. Neither E2 nor FSH was significantly associated with LBP.

Associations of gut permeability with inflammation and BMD. Median hs-CRP increased from $1.4 \mathrm{mg}$ in premenopause to $1.6 \mathrm{mg} / \mathrm{L}$ in postmenopause, but this increase did not reach statistical significance $(P=$ 0.06, Wilcoxon signed-rank test). The distribution of hs-CRP was skewed. During the same period, mean lumbar spine (LS) and total hip (TH) BMD decreased from 1.104 to $0.986 \mathrm{~g} / \mathrm{cm}^{2}$ and from 0.964 to 0.901 $\mathrm{g} / \mathrm{cm}^{2}$, respectively $(P<0.00001$ for both sites). Scatter plots of FABP2, LBP, and sCD14 versus hs-CRP are presented in Figure 1. Figure 2 contains scatter plots of FABP2, LBP, sCD14, and hs-CRP versus BMD; we present plots for LS only, as those for FN were similar (data not shown).

To test whether greater FABP2, LBP, or SCD14 is associated with greater hs-CRP, we used repeated measures, mixed-effects linear regression (Table 3). hs-CRP was log transformed for these analyses because of skewed distributions. Adjusted for age at the premenopausal visit, race/ethnicity, BMI, and study site, each SD increment in LBP and SCD14 was associated with 2.32 -fold $(P<0.001)$ and 1.44 fold $(P=0.001)$ greater hs-CRP, respectively. FABP2 was not associated with hs-CRP.

To examine the associations of FABP2, LBP, sCD14, and hs-CRP with BMD, we again used repeated measures, mixed-effects linear regression (Table 4). Adjusted for the same covariates listed above, each SD increment in FABP2 was associated with 0.021 and $0.017 \mathrm{~g} / \mathrm{cm}^{2}$ lower LS $(P=0.05)$ and TH $\operatorname{BMD}(P=0.02)$. Similarly, each SD increment in SCD14 was associated with 0.051 lower LS BMD $(P$ $=0.0001)$ and $0.025 \mathrm{~g} / \mathrm{cm}^{2}$ lower TH BMD $(P=0.004)$. LBP was not associated with BMD. In contrast, each 2-fold increment in hs-CRP was associated with $0.024 \mathrm{~g} / \mathrm{cm}^{2}$ lower LS $(P<0.0001) \mathrm{BMD}$; hs-CRP was not associated with TH BMD.

\section{Discussion}

The primary objective of this longitudinal, pilot study was to determine if gut permeability (assessed noninvasively using markers of gut barrier dysfunction [FABP2] and immune activation secondary to microbial translocation [LBP, sCD14]) increases during the MT. Upon observing this increase, our exploratory objective was to examine whether greater gut permeability was associated with greater inflammation and lower BMD. Our primary analysis supports the hypothesis that, in humans, gut permeability increases from pre- to postmenopause. Exploratory analyses suggest that greater gut permeability (LBP and sCD14) 
A
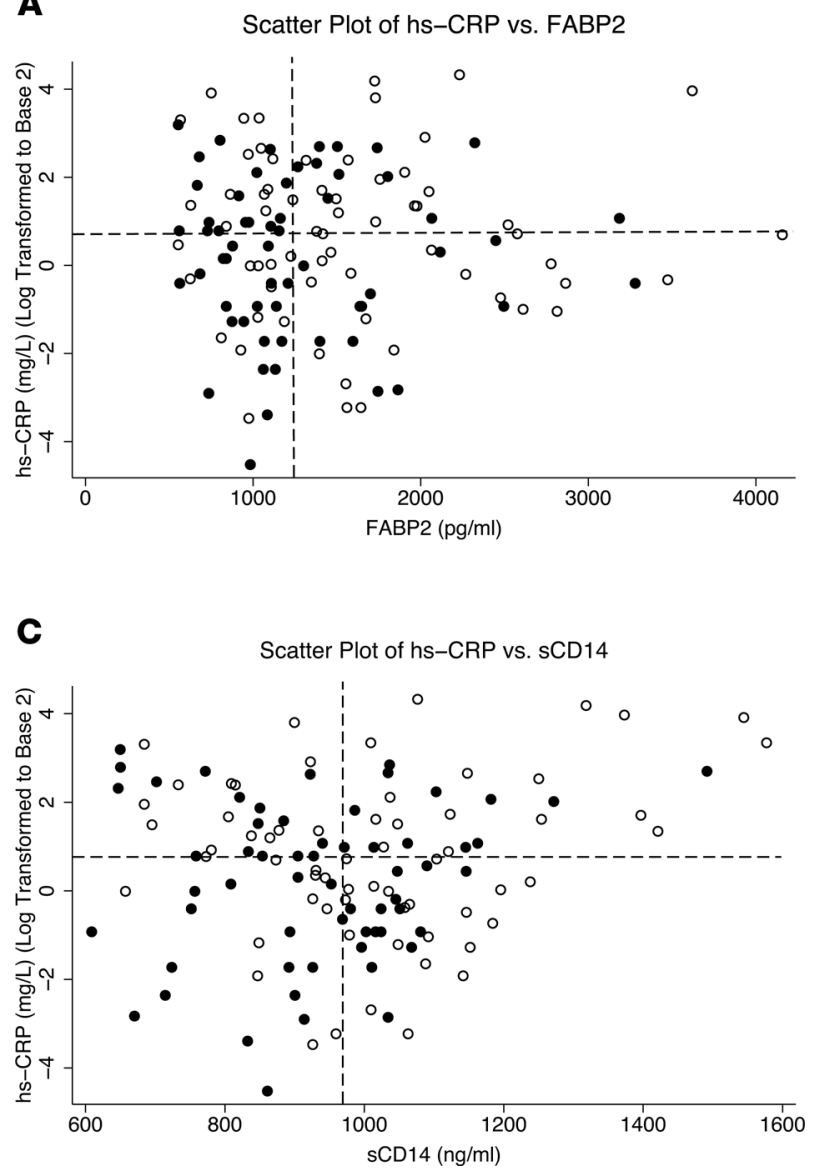

B

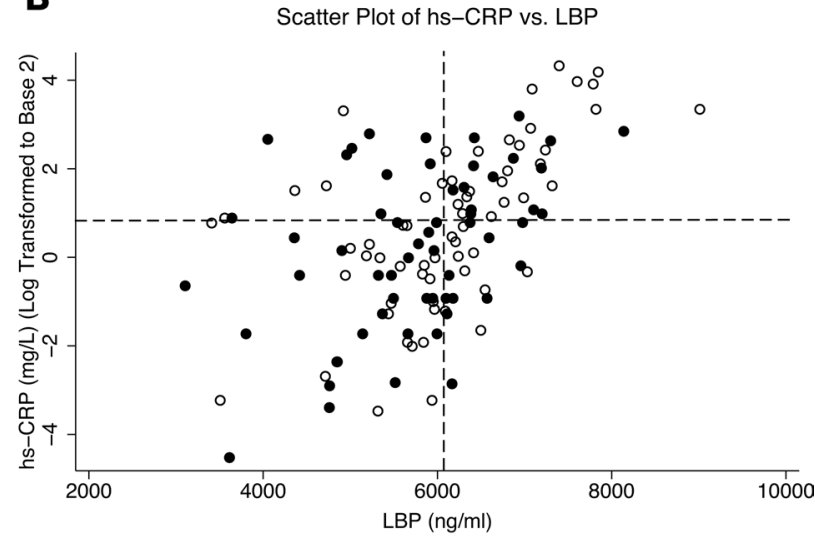

Figure 1. Scatter plots of gut permeability markers versus hs-CRP. (A-C) Plots of paired measures of FABP2 (A) (gut barrier dysfunction marker), LBP (B) (immune activation/gut microbial translocation marker), or sCD14 (C) (immune activation/gut microbial translocation marker) versus hs-CRP. Paired measures were obtained before menopause (closed circles, 3-5 years before the final menstrual period) and after menopause (open circles, 3-5 years after the final menstrual period). Vertical dashed lines indicate the median gut permeability marker values, and horizontal dashed lines mark the median hs-CRP value. A total of 65 subjects were included.

is associated with greater inflammation (hs-CRP), and both greater gut permeability and inflammation (FABP2, sCD14, hs-CRP) are associated with lower BMD.

Several prior studies have reported the effects of aging, but not the MT, on gut permeability in humans. One cross-sectional analysis found that gut permeability was higher in older versus younger men and women (20). Subsequently, the longitudinal Cardiovascular Health Study (CHS) determined that sCD14 increased by $0.4 \%$ per year in men and women over 65 years $(P<0.0001)(21)$. This is the first human study, to our knowledge, to demonstrate that gut permeability (assessed indirectly) increases during the MT. This finding is additionally supported by data showing that lower E2 and greater FSH are associated with greater FABP2 and sCD14.

In rodent models, increased gut permeability underlies an MT-related increase in inflammation. Chemical or surgical menopause leads to decreased expression of epithelial junction proteins (e.g., claudins 1, 2, and 3 and Jam3) and increased gut permeability (12). This, in turn, permits the translocation of microbes from the intestinal lumen into the subepithelial space, triggering immune cells to produce proinflammatory cytokines (12). Our exploratory analyses suggest that immune activation from gut microbial translocation (LBP and sCD14) is associated with greater inflammation (hs-CRP). The implications are that a mechanism that contributes to inflammation in rodents may similarly be present in humans and that MT-related changes in gut physiology warrants further research as a potential therapeutic target for preventing inflammation.

The physiologic importance of increased gut permeability and inflammation is highlighted in rodent studies in which probiotics that reduce gut permeability (12) or inflammation (13-15) can decrease bone resorption (12-15) and prevent bone loss $(12,15)$. In humans, a prior SWAN study reported that greater inflammation (assessed by hs-CRP) is associated with lower BMD (cross-sectional), lower hip strength (cross-sectional), and more future fractures (4). We also have ongoing analyses using the full SWAN Bone Cohort to examine the associations of change in hs-CRP and IL- 6 with change in BMD. Here, we report that greater FABP2 and sCD14 are associated with lower LS and TH BMD, and greater hs-CRP is associated with lower LS BMD. 
A
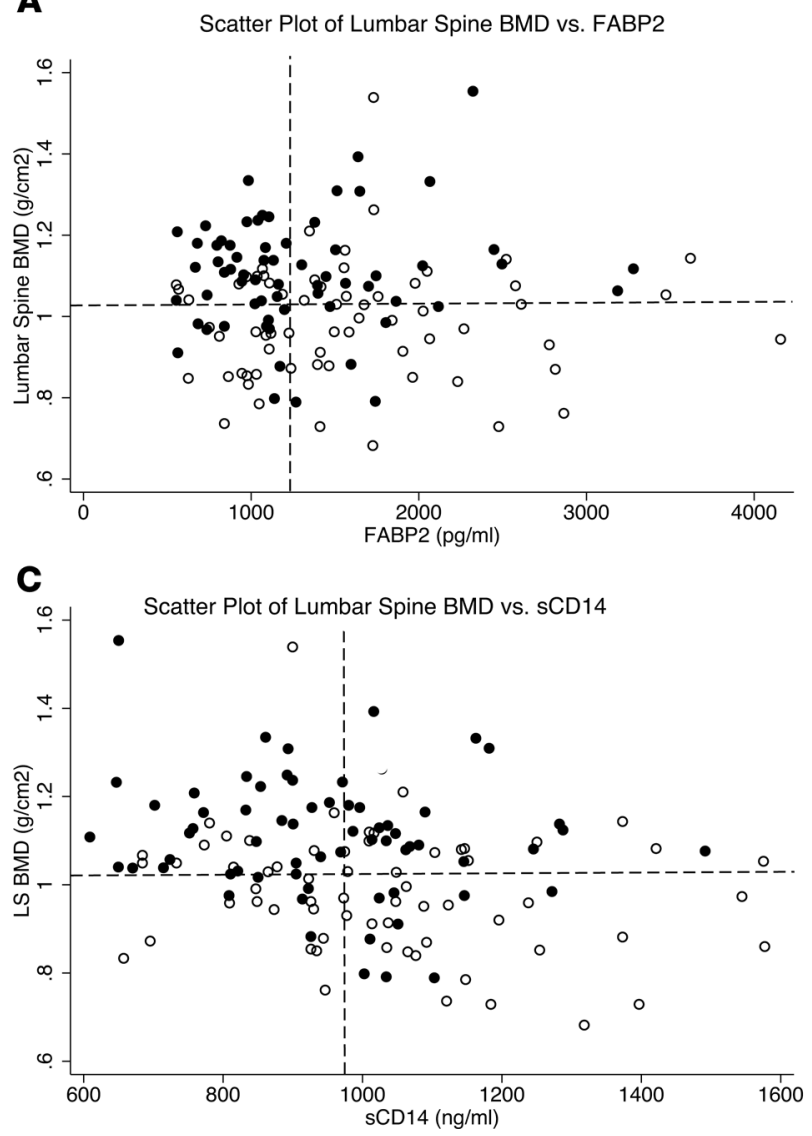

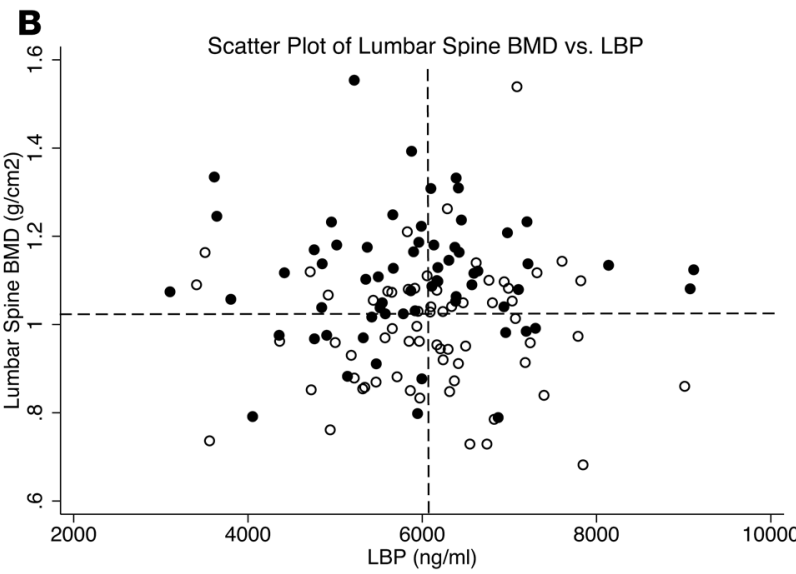

D

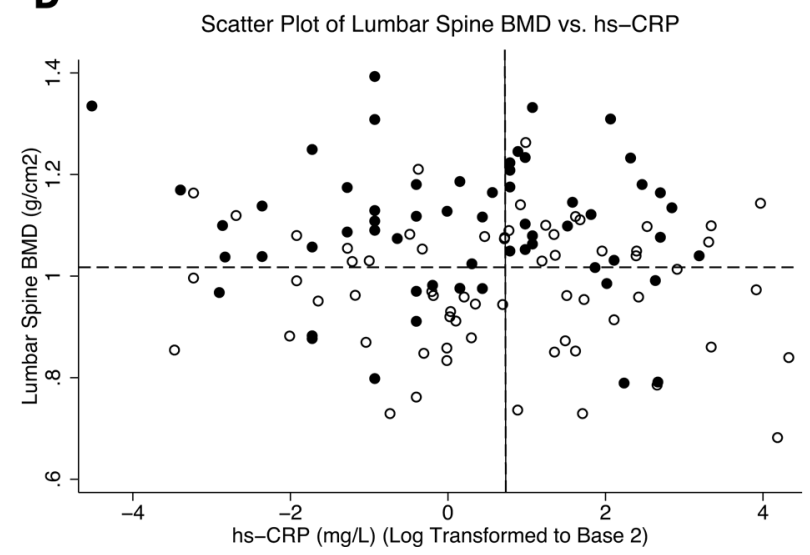

Figure 2. Scatter plots of gut permeability and inflammatory markers versus lumbar spine bone mineral density. (A-D) Plots of paired measures of FABP2 (A) (gut barrier dysfunction marker), LBP (B) (immune activation/gut microbial translocation marker), sCD14 (C) (immune activation/gut microbial translocation marker), or hs-CRP (D) (inflammatory marker) versus lumbar spine bone mineral density. Paired measures were obtained before menopause (closed circles, 3-5 years before the final menstrual period) and after menopause (open circles, 3-5 years after the final menstrual period). Vertical dashed lines indicate median gut permeability or inflammatory marker values, and horizontal dashed lines mark the median lumbar spine bone mineral density value. A total of 65 subjects were included.

While these findings suggest a link between gut permeability, inflammation, and BMD, this study was not sufficiently powered to explicitly relate changes in FABP2, LBP, sCD14, and hs-CRP to concurrent change in $\mathrm{BMD}$. We also could not discern whether inflammation versus circulating bacterial products contribute more to lower BMD.

Gut permeability can be assessed by various methods. Histologic and electron microscopic evaluation of biopsy samples provides direct information on gut barrier integrity and mucosal tight junctions (22). Alternatively, enteral administration of nondigestible markers affords a functional assessment of gut permeability (22-24). However, neither methodology is well suited to community-based cohort designs. For this pilot study, we indirectly assessed gut permeability using markers of gut barrier dysfunction (FABP2) and immune activation secondary to gut microbial translocation (LBP, sCD14). These markers are associated with adverse outcomes in pathologic conditions characterized by increased gut permeability (e.g., liver cirrhosis, inflammatory bowel disease, HIV, and sepsis) $(18,25-28)$. That FABP2, LBP, and sCD14 increase from pre- to postmenopause suggests that they are sensitive and precise enough to examine within-individual changes in gut physiology during the MT. Ideally, prior to large-scale implementation of these blood markers in observational research, their correlation with functional measurements of gut permeability using nondigestible markers should be determined.

While FABP2 is specific to the intestine (17), LBP and sCD14 are not; they are produced by hepatocytes and immune cells, respectively $(18,19,22)$. We presume that increases in LBP and sCD14 reflect immune activation from gut microbial translocation because the gut is the primary source of gram-negative microbes (29). However, the vaginal flora becomes more gram negative during postmenopause (30). 
Table 3. Associations of gut permeability ${ }^{A}$ with a marker of inflammation ${ }^{B}$

\begin{tabular}{|c|c|c|}
\hline & $\begin{array}{l}\text { "X"-fold increment in hs-CRP per SD increment in FABP2, LBP, } \\
\text { or SCD14 }(95 \% \mathrm{CI})^{c}\end{array}$ & $P$ value \\
\hline \multicolumn{3}{|c|}{ Gut barrier integrity or immune activation markers } \\
\hline FABP2 & $-0.05(-0.02,+0.02)$ & 0.5 \\
\hline LBP & $+2.32(+1.87,+2.87)$ & 0.0001 \\
\hline sCD14 & $+1.44(+1.15,+1.80)$ & 0.001 \\
\hline \multicolumn{3}{|c|}{$\begin{array}{l}\text { ACut permeability assessed by FABP2 (barrier dysfucntion), LBP, and sCD14 (immune activation secondary to gut microbial translocation). }{ }^{B} \text { Inflammation } \\
\text { assessed by hs-CRP. CAssociations assessed by repeated measured, mixed-effect linear regression with hs-CRP as continuous outcome variable, and FABP2, } \\
\text { LBP, or sCD14 as continuous primary predictors (each modeled separately). Analyses were adjusted for age at premenopausal time point, BMI, race/ethnicity, } \\
\text { and Study of Women's Health Across the Nation (SWAN) study site. }\end{array}$} \\
\hline
\end{tabular}

Thus, gram-negative microbial translocation could also occur across the vaginal epithelium. Future studies should consider whether postmenopausal loss of epithelial integrity contributes to a greater inflammatory burden.

The primary strength of this pilot is that we examined longitudinal changes in markers of gut permeability in a well-characterized sample of women transitioning through the MT. In addition, in exploratory analyses, we related these gut permeability markers to a marker of inflammation and BMD. This study is a critical first step in elucidating whether a mechanism that contributes to inflammation and disease in animal models is similarly present in humans.

The principal limitation of this study is the modest sample size of 65 women, which was, nonetheless, powered to our primary objective: to determine if gut permeability increases during the MT. Our primary analysis upheld that all tested markers of gut permeability increase from pre- to postmenopause. While our exploratory analyses suggest a link between gut permeability, inflammation, and BMD, not all predictor-outcome associations were significant. In addition, this study was not sufficiently powered to explicitly examine the longitudinal associations between within-woman changes in exposures and outcomes. Nonetheless, our pilot data provide a strong rationale to conduct future studies of these questions using larger study samples. Specifically, future investigations should explore the trajectories of change in gut permeability across the MT and relate the longitudinal changes in gut permeability and inflammation to bone loss and other end-organ manifestations. Lastly, we measured a limited number of gut permeability markers. Future work should also assay markers such as zonulin

Table 4. Associations of gut permeability ${ }^{A}$ with bone mineral density

\begin{tabular}{|c|c|c|}
\hline & $\begin{array}{l}\text { Increment in lumbar spine or total hip bone mineral density }\left(\mathrm{g} / \mathrm{cm}^{2}\right) \text { per SD increment in FABP2, } \\
\text { LBP, and sCD14, or per 2-fold increment in hs-CRP }(95 \% \mathrm{CI})^{\mathrm{B}}\end{array}$ & $P$ value \\
\hline \multicolumn{3}{|l|}{ FABP2 } \\
\hline Lumbar spine & $-0.021(-0.043,0.000)$ & 0.05 \\
\hline Total hip & $-0.017(-0.031,-0.003)$ & 0.02 \\
\hline \multicolumn{3}{|l|}{ LBP } \\
\hline Lumbar spine & $-0.018(-0.047,0.010)$ & 0.2 \\
\hline Total hip & $-0.005(-0.239,0.015)$ & 0.6 \\
\hline \multicolumn{3}{|l|}{ sCD14 } \\
\hline Lumbar spine & $-0.051(-0.076,-0.026)$ & 0.0001 \\
\hline Total hip & $-0.025(-0.041,-0.008)$ & 0.004 \\
\hline \multicolumn{3}{|l|}{ hs-CRP } \\
\hline Lumbar spine & $-0.024(-0.038,-0.011)$ & $<0.0001$ \\
\hline Total hip & $-0.008(-0.018,0.002)$ & 0.1 \\
\hline \multicolumn{3}{|c|}{$\begin{array}{l}\text { ACut permeability assessed by FABP2 (barrier dysfucntion), LBP, and sCD14 (immune activation secondary to gut microbial translocation). }{ }^{B} \text { Associations assessed } \\
\text { by repeated measured, mixed-effect linear regression with bone mineral density at the lumbar spine or femoral as continuous outcome variables, and FABP2, } \\
\text { LBP, or sCD14 as continuous primary predictors (each modeled separately). Analyses were adjusted for age at premenopausal time point, BMI, race/ethnicity, and } \\
\text { Study of Women's Health Across the Nation (SWAN) study site. }\end{array}$} \\
\hline
\end{tabular}




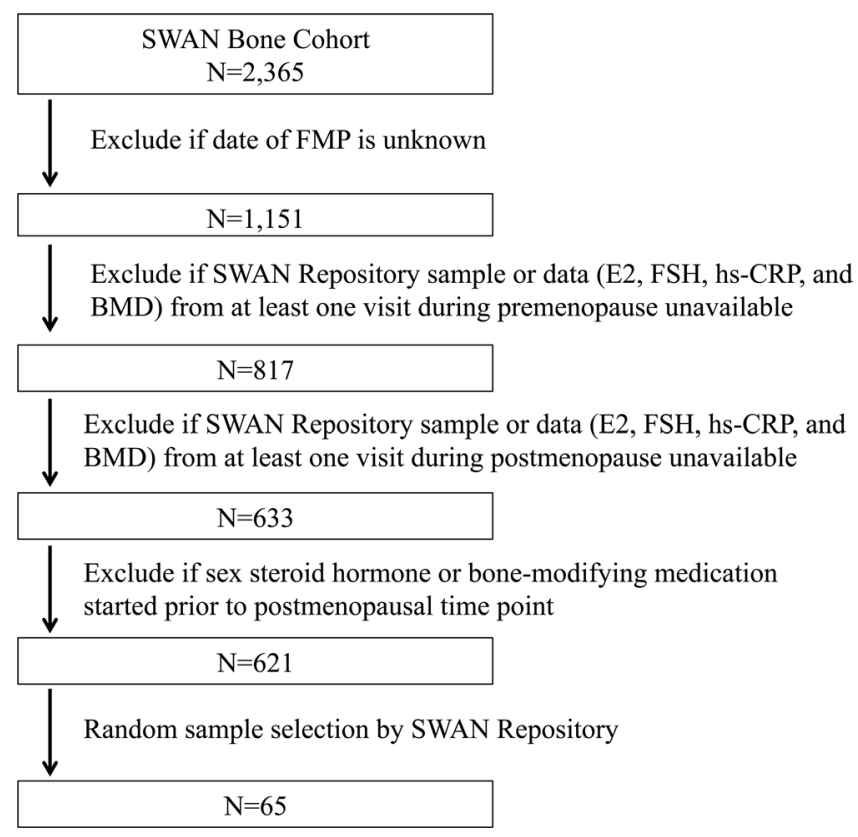

Figure 3. Analysis sample derivation. This flow chart shows the derivation of the analysis sample. In order to be included in the study, participants needed to meet the following criteria: (a) have undergone natural menopause; (b) have a known final menstrual period (FMP) date; (c) have at least 1 visit in premenopause and 1 visit in postmenopause from which previously collected plasma could be accessed through the SWAN Repository to measure FABP2, LBP, and SCD14; (d) have preexisting measures of E2, FSH, hs-CRP, and BMD corresponding to the plasma sample times; and (e) have not used sex steroid medications or bone-modifying agents in between their pre- and postmenopausal pilot study visits. A total of 621 women met these criteria. From this potential pool of subjects, the SWAN Repository randomly selected 65 women for this pilot study.

and caludin 3, which reflect gut epithelial paracellular integrity $(31,32)$, since decreased estrogen downregulates gut epithelial junction proteins (12).

In summary, our findings support that gut permeability increases from pre- to postmenopause, and exploratory results suggest a relation between gut permeability, inflammation, and BMD that should be further investigated in larger study samples. To our knowledge, this is the first demonstration of a MT-related "gut leak" in humans. If, indeed, increased gut permeability during the MT leads to a state of immune activation and inflammation with negative health consequences, the clinical implications could be substantial: interventions that target gut physiology during and after the MT could lessen inflammation and multiple disorders that plague older adults.

\section{Methods}

\section{SWAN}

SWAN, the parent study for this pilot, is a multicenter, longitudinal cohort study of the MT in 3,302 ambulatory, multiracial/ethnic (Black, Chinese, Japanese, White) women. The SWAN cohort has been described in detail (33). In brief, at SWAN baseline, participants were in premenopause (no change in menstrual bleeding in the past year) or early perimenopause (less predictable menstrual bleeding at least once every 3 months), and between 42 and 52 years of age. After 2 decades of observational follow-up, consisting of 17 serial visits, all SWAN women have transitioned to postmenopause. To access BMD data, we selected our pilot sample from the SWAN Bone Cohort, a subset of 2365 participants, in whom BMD was measured at each study visit. The SWAN Repository provided previously collected and frozen plasma samples to measure the various markers of gut permeability $(33,34)$.

\section{Study sample}

Eligibility. To be included in the pilot, participants were required to: (a) have undergone natural menopause; (b) have a known FMP date; (c) have at least 1 visit in premenopause (operationalized as having occurred 3-5 years prior to the FMP) and 1 visit in postmenopause (operationalized as having occurred between 3-5 years 
after the FMP) from which previously collected plasma could be accessed through the SWAN Repository to measure FABP2, LBP, and sCD14; (d) have preexisting measures of E2, FSH, hs-CRP, and BMD corresponding to the plasma sample times; and (e) have not used sex steroid medications or bone modifying agents between their pre- and postmenopausal pilot study visits. We selected the timing of the pre- and postmenopausal samples (relative to the FMP date) based on the trajectories of change in estradiol across the MT; estradiol does not decreases significantly until 3 years before the FMP and nadirs at about 3 years after the FMP (35).

Sample derivation. Figure 3 depicts the derivation of the analysis sample. In total, 621 women met our eligibility criteria. From this potential pool of subjects, the SWAN Repository randomly selected 65 women for this pilot study (see Analysis for sample size considerations).

\section{Phlebotomy}

Every effort was made to perform phlebotomy fasting and performed before 10 am during the early follicular phase (EFP, between days 2 and 5) of a spontaneous menstrual cycle (prior to postmenopause). If an EFP sample could not be obtained after 2 attempts, a random fasting sample was taken within a 90-day window of the anniversary of the baseline visit. Collected specimens were initially stored between $-20^{\circ} \mathrm{C}$ and $-80^{\circ} \mathrm{C}$ at individual study sites for up to 30 days; they were then shipped to a central lab (either Medical Research Lab [Highland Heights, Kentucky, USA] or CLASS Laboratory at the University of Michigan [Ann Arbor, Michigan, USA]). Once at the central lab, samples were stored at $-80^{\circ} \mathrm{C}$.

\section{Marker of gut permeability}

To assess gut permeability, we measured markers of gut barrier dysfunction (FABP2) and immune activation secondary to microbial translocation (LBP, sCD14) using citrated plasma samples that were accessed from the SWAN Repository. FABP2 was measured using the Quantikine Human FABP2/I-FABP ELISA (R\&D Systems). The lower limit of detection (LLD) for this assay is $6.21 \mathrm{pg} / \mathrm{mL}$. The intraassay coefficient of variation $(\mathrm{CV})$ was $<4.1 \%$. LBP and SCD14 were assayed in multiplex, using the Luminex platform with custom-made panels ( $R \& D$ Systems). This platform uses microparticles that are precoated with analyte-specific antibodies and incubated with diluted plasma samples, followed by a biotin antibody and by a streptavidin-phycoreythin conjugate. The fluorescence intensity of each analyte's microparticles are then quantified using a Bioplex 200 (Luminex) System Analyzer, and the data are analyzed using Bioplex Manager software. The LLD for the LBP is $0.0839 \mathrm{ng} / \mathrm{mL}$, and the intra-assay CV was $<2.9 \%$. The LLD for $\mathrm{sCD} 14$ is $0.0366 \mathrm{ng} / \mathrm{mL}$, and the intra-assay $\mathrm{CV}$ was $<2.9 \%$.

\section{Markers of ovarian function}

To examine whether reduced ovarian function is associated with greater gut permeability across the MT, we used previously measured E2 and FSH. Serum E2 was measured in duplicate with a modified, off-line ACS:180 (E2-6) immunoassay using an ACS:180 automated analyzer (Bayer Diagnostics Corp.). The average between duplicates was recorded in the dataset and used in analyses. The LLD was $1.0 \mathrm{pg} / \mathrm{mL}$, and the intraassay CV was $6.4 \%$. Serum FSH was measured in singlicate with a 2-site chemiluminometric assay (Bayer Diagnostics Corp.). The LLD was $1.05 \mathrm{mIU} / \mathrm{mL}$, and intraassay CV was $6.0 \%$. For this pilot, we only included women whose premenopausal E2 and FSH measurements were obtained in the EFP of a spontaneous menstrual cycle.

\section{hs-CRP}

To assess whether the associations of gut permeability with inflammation, and of inflammation with BMD, we used hs-CRP. CRP was measured at SWAN baseline and at follow-up visits 1, 3-7, 9, 10, 12, and 15. Owing to the long duration of follow-up, the laboratories that measured CRP and the assays employed changed with time. SWAN used 3 CRP assays and a fourth, hs-CRP assay as the calibration standard to harmonize all assays. The fourth assay was also used to obtain results for analytes that had been below the LLD on each of the 3 original assays. From baseline to follow-up visit 7, CRP was measured by Medical Research Laboratories. CRP assays for samples collected at follow-up visit 9 and later were conducted by the CLASS Laboratory at the University of Michigan using the Alfa Wassermann ACE analyzer. Assays and their calibration are described below.

CRP assay 1. From baseline to follow-up visit 7, CRP was measured using an ultrasensitive rate immunonephelo-metric method, with a LLD of $0.3 \mathrm{mg} / \mathrm{L}$ (BN100; Dade-Behring). The intraassay CV at CRP concentrations of 0.5 and $22.0 \mathrm{mg} / \mathrm{L}$ were $10 \%-12 \%$ and $5 \%-7 \%$, respectively. 
CRP assay 2. For follow-up visit 12, CRP was assayed using the ACE UltraWide Range assay, a latex-enhanced turbidimetric in vitro immunoassay (Alfa Wassermann). The LLD was $0.1 \mathrm{mg} / \mathrm{L}$, and the intraassay CV at CRP concentrations of $0.5 \mathrm{mg} / \mathrm{L}$ and $9.8 \mathrm{mg} / \mathrm{L}$ were $5.7 \%-7.0 \%$ and $1.2 \%$, respectively.

CRP assay 3. Samples from follow-up visits 9,10 , and 15 were assayed using a high-sensitivity immunoassay (Alfa Wassermann). The LLD was $0.1 \mathrm{mg} / \mathrm{L}$, and the intraassay CV at CRP concentrations of 0.5 and $9.8 \mathrm{mg} / \mathrm{L}$ were $5.7 \%-7.0 \%$ and $1.2 \%$, respectively.

CRP assay 4. For approximately $25 \%$ of all samples run between baseline and follow-up visit 15 , CRP was below the LLD of the original 3 assays outlined above. In these instances, an additional sample was retrieved from the SWAN Repository, and CRP was measured using the Human High Sensitivity CRP ELISA (R\&D Systems, DCRP00), a quantitative sandwich enzyme immunoassay. The lower LLD for this assay was 0.10 $\mathrm{pg} / \mathrm{mL}$. The remaining $75 \%$ of results (those above the LLD for the 3 original assays) were calibrated to the high-sensitivity ELISA by simultaneously assaying 600 paired samples (representing the full range of results from each of the original 3 assays) and the high-sensitivity assay; thus, there were 200 paired samples for each original versus high-sensitivity ELISA. After calibration, correlations between the first 3 CRP assays and the fourth, high-sensitivity ELISA were $\geq 0.94$.

\section{BMD}

BMD at the LS and TH BMD was measured by DXA. At study inception, the Pittsburgh (Pennsylvania, USA) and Oakland (California, USA) sites used the Hologic QDR 2000 machine, and the Boston (Massachusetts, USA), Los Angeles (California, USA), and Ann Arbor (Michigan, USA) sites used the Hologic QDR 4500A machine. At follow-up visit 8, Pittsburgh and Oakland upgraded to the 4500A models. Boston and Los Angeles upgraded to the Hologic Discovery model at follow-up visit 13 . To develop cross-calibration regression equations, each site obtained duplicate scans using the old and new hardware within 90 days in at least 40 volunteers ( $n=40$ for Oakland, Pittsburgh; $n=41$ for Boston; and $n=50$ for Los Angeles). To determine the short-term in vivo precision error, each study site measured LS and TH BMD twice in 5 women with complete subject repositioning between duplicate scans. Using the root mean square SD approach, the precision error in SWAN was $1.4 \%$ at the LS and $2.2 \%$ at the TH. An anthropomorphic spine phantom was circulated between sites for cross-site calibration. Standard quality control phantom scans were conducted before each BMD measurement session. If necessary, these were used to adjust for longitudinal machine drift.

\section{Additional measures and variables}

The following were included in analyses as covariates: age (years), race/ethnicity (Black, Chinese, Japanese, White), and BMI (weight in kilograms/[height in meters $]^{2}$ ), based on height and weight measured using standardized protocols and with participants wearing light clothing and no shoes.

\section{Statistical analyses}

Sample size. The sample size of 65 subjects was predicated on funding constraints and the power to detect change in our primary outcomes. Because there are no published cross-sectional or longitudinal studies examining whether gut permeability (assessed directly or indirectly) increase across the MT, we relied on change in sCD14 with aging published in the CHS (21). In CHS, sCD14 increased by $0.4 \%$ per year (21), which would result in a gain of $2.4 \%-4.0 \%$ over a 6 - to 10 -year period (the approximate time interval between our pre- to postmenopausal time points). A sample size of 65 yielded $90 \%$ power at a 2 -sided $\alpha$ of 0.05 to detect $4.5 \%$, $4.4 \%$, and $2.4 \%$ changes in FABP2, LBP, and SCD14, respectively, using the interassay CV for each analyte.

Data analyses. We generated descriptive statistics for all variables and assessed the distributions of continuous variables for normality. E2, FSH, and hs-CRP had skewed distributions and were, thus, log-transformed for relevant analyses. For all analyses, a $P$ value of $\leq 0.05$ was considered to be statistically significant.

To assess our primary hypothesis, we used the paired, 2-tailed $t$ test at a 2-sided $\alpha$ of 0.05 to test the mean within-person change in FABP2, LBP, and SCD14 from pre- to postmenopause against the null hypothesis of zero change. We considered a $P$ value of 0.05 and lower to be statistically significant. To additionally examine whether ovarian function is associated with gut permeability, we used repeated measures, mixed-effects linear regression, with E2 or FSH as continuous primary predictors and FABP2, LBP, or SCD14 as continuous outcome measures (with each primary predictor-outcome pair modeled separately). Models included a random intercept at the individual level to account for clustering. Covariates were chronologic age at the premenopausal visit, BMI, race/ethnicity, and SWAN study site. 
To test our secondary hypothesis, we used repeated measures, mixed-effects linear regression with FABP2, LBP, sCD14, or hs-CRP as continuous primary predictors and hs-CRP, LS BMD, or TH BMD as continuous outcomes variables. As above, each primary predictor-outcome pair was modeled separately, and all models included a random intercept at the individual level. Covariates were chronologic age at the premenopausal visit, BMI, race/ethnicity, and study site.

For all relational analyses stipulated above, we used mixed-effects, repeated measures linear regression because this approach is best suited for detecting predictor-outcome associations in small samples. This is because this type of model accounts for the associations of both between-women and within-woman differences in the predictor with corresponding differences in the outcome.

\section{Study approval}

Each SWAN clinical site obtained IRB approval (University of Pittsburgh, Pittsburgh, Pennsylvania, USA; Kaiser Permanente, Bedford, Ohio, USA; Massachusetts General Hospital, Boston, Massachusetts, USA; UCLA; and University of Michigan), and all participants provided written informed consent.

\section{Author contributions}

Participant recruitment for the parent SWAN study was contributed by GAG. Data management and cleaning were contributed by AS, ASK, and GAG. Performance of gut permeability assays was contributed by ME. Analytic design and statistical analysis were contributed by AS, ASK, and GAG. Primary manuscript drafting was contributed by AS and GAG. Critical review and revision of manuscript were contributed by AS, ME, ASK, and GAG.

\section{Acknowledgments}

SWAN has grant support from the NIH, DHHS, through the National Institute on Aging (NIA), the National Institute of Nursing Research (NINR), and the NIH Office of Research on Women's Health (ORWH) (grants U01NR004061, U01AG012505, U01AG012535, U01AG012531, U01AG012539, U01AG012546, U01AG012553, U01AG012554, and U01AG012495). The content of this article is solely the responsibility of the authors and does not necessarily represent the official views of the NIA, NINR, ORWH, or the NIH. We thank the all the women who participated in SWAN, Michelle Liao for figure preparation, and the study staff at each site.

Clinical Centers include University of Michigan (Siobán Harlow, PI 2011-present; MaryFran Sowers, PI 1994-2011); Massachusetts General Hospital (Joel Finkelstein, PI 1999-present; Robert Neer, PI 1994-1999); Rush University, Rush University Medical Center (Howard Kravitz, PI 2009-present; Lynda Powell, PI 1994-2009); University of California, Davis/Kaiser (Ellen Gold, PI); UCLA (Gail Greendale, PI); Albert Einstein College of Medicine (Carol Derby, PI 2011-present; Rachel Wildman, PI 2010-2011; Nanette Santoro, PI 2004-2010); University of Medicine and Dentistry - New Jersey Medical School (Gerson Weiss, PI 1994-2004); and the University of Pittsburgh (Karen Matthews, PI).

The NIH Program Office was operated by the National Institute on Aging (Bethesda, Maryland, USA) and the National Institute of Nursing Research (Bethesda, Maryland, USA). Program Officers included Chhanda Dutta (2016-present); Winifred Rossi (2012-2016); Sherry Sherman (1994-2012); and Marcia Ory (1994-2001). The Central Laboratory was operated by the University of Michigan in Ann Arbor (Daniel McConnell, Central Ligand Assay Satellite Services). The Coordinating Center is currently operated by the University of Pittsburgh (Maria Mori Brooks, PI 2012-present; Kim Sutton-Tyreell, PI 2001-2012), and previously operated by New England Research Institutes (Watertown, Massachusetts, USA; Sonja McKinlay, PI 1995-2001).

The Current Chair of the Steering Committee is Susan Johnson. Chris Gallagher was the Former Chair. This report is based on samples from the SWAN Core Repository. Scientists interested in developing studies based on this resource can find a description of the SWAN Core Repository and SWAN DNA Repository and information on obtaining access to the resources at http://www.nia.nih.gov/research/dgcg/ study-womens-health-across-nation-swan-repository. 
Address correspondence to: Albert Shieh, UCLA Division of Geriatrics, 10945 Le Conte Avenue, Suites 23392345, Los Angeles, California 90095-1687, USA. Phone: 781.254.5034; Email: ashieh@mednet.ucla.edu.

1. Kennedy BK, et al. Geroscience: linking aging to chronic disease. Cell. 2014;159(4):709-713.

2. Prasad S, Sung B, Aggarwal BB. Age-associated chronic diseases require age-old medicine: role of chronic inflammation. Prev Med. 2012;54 Suppl:S29-S37.

3. Franceschi C, Garagnani P, Parini P, Giuliani C, Santoro A. Inflammaging: a new immune-metabolic viewpoint for age-related diseases. Nat Rev Endocrinol. 2018;14(10):576-590.

4. Ishii S, et al. C-reactive protein, bone strength, and nine-year fracture risk: data from the Study of Women's Health Across the Nation (SWAN). J Bone Miner Res. 2013;28(7):1688-1698.

5. Wang NC, Matthews KA, Barinas-Mitchell EJ, Chang CC, E1 Khoudary SR. Inflammatory/hemostatic biomarkers and coronary artery calcification in midlife women of African-American and White race/ethnicity: the Study of Women's Health Across the Nation (SWAN) heart study. Menopause. 2016;23(6):653-661.

6. Ding C, Parameswaran V, Udayan R, Burgess J, Jones G. Circulating levels of inflammatory markers predict change in bone mineral density and resorption in older adults: a longitudinal study. J Clin Endocrinol Metab. 2008;93(5):1952-1958.

7. $\mathrm{Xu} \mathrm{H}$, et al. Chronic inflammation in fat plays a crucial role in the development of obesity-related insulin resistance. $J$ Clin Invest. 2003;112(12):1821-1830.

8. Ridker PM, Hennekens CH, Buring JE, Rifai N. C-reactive protein and other markers of inflammation in the prediction of cardiovascular disease in women. NEngl J Med. 2000;342(12):836-843.

9. Yaffe $\mathrm{K}$, et al. The metabolic syndrome, inflammation, and risk of cognitive decline. JAMA. 2004;292(18):2237-2242.

10. Woodard GA, et al. C-reactive protein is associated with aortic stiffness in a cohort of African American and white women transitioning through menopause. Menopause. 2011;18(12):1291-1297.

11. Baylis D, Bartlett DB, Patel HP, Roberts HC. Understanding how we age: insights into inflammaging. Longev Healthspan. 2013;2(1):8

12. Li JY, et al. Sex steroid deficiency-associated bone loss is microbiota dependent and prevented by probiotics. J Clin Invest. 2016;126(6):2049-2063.

13. Ohlsson C, et al. Probiotics protect mice from ovariectomy-induced cortical bone loss. PLoS ONE. 2014;9(3):e92368.

14. Britton RA, et al. Probiotic L. reuteri treatment prevents bone loss in a menopausal ovariectomized mouse model. J Cell Physiol. 2014;229(11):1822-1830.

15. Collins FL, et al. Lactobacillus reuteri 6475 Increases Bone Density in Intact Females Only under an Inflammatory Setting. PLoS ONE. 2016;11(4):e0153180.

16. Pelsers MM, et al. Intestinal-type and liver-type fatty acid-binding protein in the intestine. Tissue distribution and clinical utility. Clin Biochem. 2003;36(7):529-535.

17. Stevens BR, et al. Increased human intestinal barrier permeability plasma biomarkers zonulin and FABP2 correlated with plasma LPS and altered gut microbiome in anxiety or depression. Gut. 2018;67(8):1555-1557.

18. Epeldegui M, et al. A prospective study of serum microbial translocation biomarkers and risk of AIDS-related non-Hodgkin lymphoma. AIDS. 2018;32(7):945-954.

19. Pugin J, Schürer-Maly CC, Leturcq D, Moriarty A, Ulevitch RJ, Tobias PS. Lipopolysaccharide activation of human endothelial and epithelial cells is mediated by lipopolysaccharide-binding protein and soluble CD14. Proc Natl Acad Sci USA. 1993;90(7):2744-2748.

20. Man AL, et al. Age-associated modifications of intestinal permeability and innate immunity in human small intestine. Clin Sci. 2015;129(7):515-527.

21. Reiner AP, et al. Soluble CD14: genomewide association analysis and relationship to cardiovascular risk and mortality in older adults. Arterioscler Thromb Vasc Biol. 2013;33(1):158-164.

22. Wang L, Llorente C, Hartmann P, Yang AM, Chen P, Schnabl B. Methods to determine intestinal permeability and bacterial translocation during liver disease. J Immunol Methods. 2015;421:44-53.

23. Peeters $\mathrm{M}$, et al. Test conditions greatly influence permeation of water soluble molecules through the intestinal mucosa: need for standardisation. Gut. 1994;35(10):1404-1408.

24. Mattioli F, et al. Assessment of intestinal permeability using sugar probes: influence of urinary volume. Clin Lab. 2011;57(11-12):909-918.

25. Sarikaya M, Ergül B, Doğan Z, Filik L, Can M, Arslan L. Intestinal fatty acid binding protein (I-FABP) as a promising test for Crohn's disease: a preliminary study. Clin Lab. 2015;61(1-2):87-91.

26. Pastor Rojo O, López San Román A, Albéniz Arbizu E, de la Hera Martínez A, Ripoll Sevillano E, Albillos Martínez A. Serum lipopolysaccharide-binding protein in endotoxemic patients with inflammatory bowel disease. Inflamm Bowel Dis. 2007;13(3):269-277.

27. Reiberger T, et al. Non-selective betablocker therapy decreases intestinal permeability and serum levels of LBP and IL-6 in patients with cirrhosis. J Hepatol. 2013;58(5):911-921.

28. Opal SM, et al. Relationship between plasma levels of lipopolysaccharide (LPS) and LPS-binding protein in patients with severe sepsis and septic shock. J Infect Dis. 1999;180(5):1584-1589.

29. Turnbaugh PJ, Ley RE, Hamady M, Fraser-Liggett CM, Knight R, Gordon JI. The human microbiome project. Nature. 2007;449(7164):804-810.

30. Pabich WL, Fihn SD, Stamm WE, Scholes D, Boyko EJ, Gupta K. Prevalence and determinants of vaginal flora alterations in postmenopausal women. J Infect Dis. 2003;188(7):1054-1058.

31. Fasano A. Intestinal permeability and its regulation by zonulin: diagnostic and therapeutic implications. Clin Gastroenterol Hepatol. 2012;10(10):1096-1100.

32. Patel RM, Myers LS, Kurundkar AR, Maheshwari A, Nusrat A, Lin PW. Probiotic bacteria induce maturation of intestinal 
claudin 3 expression and barrier function. Am J Pathol. 2012;180(2):626-635.

33. Sowers M, et al. In: Lobo R, Kelsey J, Marcus R eds. Menopause: Biology and Pathobiology. San Diego, California, USA: Academic Press; 2000:175-88.

34. Kardia SR, Chu J, Sowers MR. Characterizing variation in sex steroid hormone pathway genes in women of 4 races/ethnicities: the Study of Women's Health Across the Nation (SWAN). Am J Med. 2006;119(9 Suppl 1):S3-15.

35. Randolph JF, et al. Change in follicle-stimulating hormone and estradiol across the menopausal transition: effect of age at the final menstrual period. J Clin Endocrinol Metab. 2011;96(3):746-754. 